\title{
Gaussian Mixture Model Learning for Multipath Assisted Positioning
}

\author{
Rostislav Karásek ${ }^{* \dagger}$, Christian Gentner* \\ *Institute of Communications and Navigation, German Aerospace Center (DLR), Wessling, Germany, rostislav.karasek@dlr.de \\ $\dagger^{\dagger}$ Department of Electromagnetic Field, Faculty of Electrical Engineering, Czech Technical University in Prague, Prague, Czechia
}

\begin{abstract}
The wireless signal distortion decreases the precision of the estimated position. However, the distortion caused by the multipath propagation was recently shown not to decrease but even improve the precision when utilized correctly. This approach is called mutipath assisted positioning. In this paper, we propose a particle filter resampling algorithm for multipath assisted positioning exploring high likelihood areas allowing a better approximation of the posterior probability density function. Thanks to the posterior probability density function modeled as a Gaussian mixture model, we can perform the exploration with the same computational load as a regularized particle filter. The proposed algorithm allows decreasing the number of particles orderly while preserving the state of the art approach's precision. We show a comparison of the state of the art ChannelSLAM algorithm with the proposed Gaussian mixture modelbased method demonstrating the significance of the achieved improvement.
\end{abstract}

Index Terms-Channel-SLAM, Gaussian mixture model, navigation, multipath assisted positioning, particle filters.

\section{INTRODUCTION}

The increasing need for precise positioning in areas unfavorable for mainstream localization systems like a GPS, based on a Line-of-sight (LoS) wireless signal propagation, gave birth to a family of multipath assisted positioning methods. These methods use an idea of turning the disadvantage of a signal distortion into an advantage. Thus, it can reliably estimate the position even when the LoS propagation is not available or is highly distorted by multipath propagation. The multipath assisted positioning methods separates into a fingerprinting [1], [2], feature mapping [3]-[5], and Simultaneous Localization And Mapping (SLAM) [6]-[10].

Due to the system's nonlinearity, the SLAM methods for multipath assisted positioning use, in most cases, sampling particles to approximate the true posterior Probability Density Function (PDF). The two main techniques practically used are Rao-Blackwellized Particle Filter (RBPF) [11], [12], and Nonparametric Belief Propagation (NBP) [13]. The disadvantage of particle-based approaches for parameter estimation is a significant computational complexity, which can be easily forbidding the algorithm to work in a real-time scenario. The number of particles used for a PDF approximation is the main parameter influencing the computational complexity. One can decrease the number of particles to speed up the algorithm while reducing precision. However, after some point, the number of particles is too small to represent the desired PDF sufficiently, and the estimation algorithm quickly fails.
We propose a method that allows us to use an extremely low number of particles to represent a complicated multimodal PDF of the Virtual Transmitter (VT) using a Gaussian Mixture Model (GMM). [14] The idea is to use an effective exploration method to increase the PDF approximation precision, improving the scaling of the RBPF for a low number of particles. The advantage of the presented exploration algorithm is that it does not increase the computational complexity than the state-of-the-art Channel-SLAM algorithm [10]. We achieve it by releasing and reusing resources during the systematic resampling [15] step of the Particle Filter (PF).

The article is structured as follows. Section II describes and defining the algorithms necessary for understanding the presented method. Section III explains the main idea of the releasing resources while performing resampling and reusing them for exploring the relevant PDF regions. Section IV compares the performance of the proposed method with the stateof-the-art approach to multipath assisted positioning. Finally, the article concludes in Section V.

\section{ALGORITHM FOUNDATIONS}

\section{A. Multipath Assisted Positioning}

The classical positioning approaches based on Time of Arrival (ToA) assume only the LoS propagation between the transmitter and receiver. However, the received signal usually consists of the transmitted signal and the delayed replicas of the transmitted signal caused by reflection and scattering. When the received signal consists of more than the LoS signal, we refer to this radio propagation channel as a multipath channel. Each replica of the transmitted signal, including the LoS, is referred to as a Multipath Component (MPC).

We can describe the multipath channel mathematically as a causal time-variant analog filter defined by its Channel Impulse Response (CIR). According to [16], the CIR can be assumed to be time-invariant for a short time interval $\delta_{t}=T_{t+1}-T_{t}$ and can be defined as a discrete function at timestep $t$ as

$$
h_{t}(\tau)=\sum_{\ell=1}^{N_{t}} \alpha_{\ell t} \cdot \delta\left(\tau-\tau_{\ell t}\right),
$$

where $N_{t}$ is the number of MPCs, $\tau_{\ell t}$, and $\alpha_{\ell t}$ are the delay and complex amplitude of the $\ell$-th MPC at time $t$. Finally, $\delta(\cdot)$ stands for the Dirac distribution. 


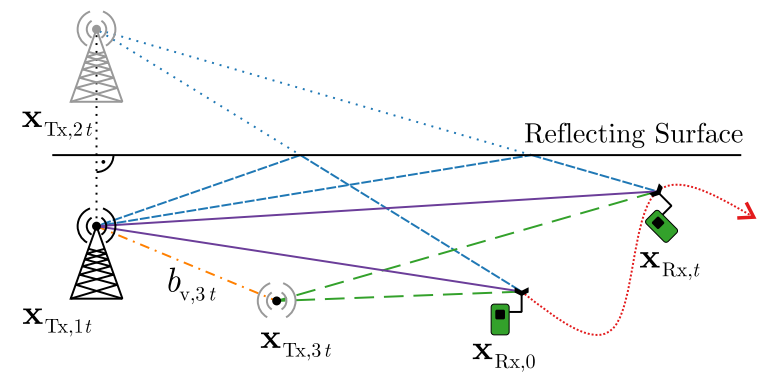

Fig. 1. Solid purple lines show the LoS between the physical transmitter $\mathbf{x}_{\mathrm{Tx}, 1 t}$, and user trajectory for two different time instances $\mathbf{x}_{\mathrm{Rx}, 0}$, and $\mathbf{x}_{\mathrm{Rx}, t}$ The MPCs caused by a reflection on a reflecting surface can be interpreted as an LoS signal propagated from $\mathrm{VT}_{2}$ at position $\mathbf{x}_{\mathrm{Tx}, 2 t}$. The position of $\mathbf{x}_{\mathrm{Tx}}, 2 t$ is obtained by mirroring $\mathbf{x}_{\mathrm{Tx}}, 1 t$ along the reflecting surface. The blue dashed line shows the real ray-like propagation, while the blue dotted line shows the VT interpretation. Additionally, the transmitted signal is scattered, and $\mathrm{VT}_{3}$ defines the position of the scatterer $\mathbf{x}_{\mathrm{Tx}}, 3 t$. The corresponding MPC delay is defined as an LoS shown by the green dashed lines, with an additional propagation distance $b_{\mathrm{v}, 3} t$ shown by the orange dash-dotted line.

The classical positioning approaches use the LoS MPC while treating all Non-line-of-sight (NLoS) MPCs as interference, decreasing the ToA-based position estimation precision. Contrary to this approach, the multipath assisted positioning tries to exploit the NLoS MPCs rather than suppress them.

Our work is based on the Channel-SLAM algorithm introduced in [8]-[10]. It uses the concept of VTs, where each of the MPCs can be modeled as an LoS signal propagating from the VT to a user carrying the receiver. Fig. 1 explains this concept, where the physical transmitter $\mathbf{x}_{\mathrm{Tx}, 1 t}$ is mirrored along the reflecting surface, creating $\mathbf{x}_{\mathrm{Tx}, 2 t}$. On top of the VT concept assumed in [7], we use a signal scattering. We can model a scatterer as a VT $\mathbf{x}_{\mathrm{Tx}, 3 t}$ placed at the position of the scatterer. However, we need to assume an additional propagation distance $b_{\mathrm{v}, 3 t}$, which corresponds to the physical transmitter and the scatterer distance. The Channel-SLAM algorithm needs no information about the floor plan and position of the VTs. Using a SLAM approach, the ChannelSLAM algorithm estimates the user position and VT position simultaneously.

\section{B. Channel-SLAM With Stochastic Data Association}

The Channel-SLAM with stochastic data association algorithm [10] assumes stochastic description, where the system state $\mathbf{x}_{0: t}$ in timesteps $1,2, \ldots, t$ is estimated jointly with the data association variable $\mathbf{n}_{1: t}$ associating the measured delays of MPCs $\mathbf{z}_{1: t}=\left[\boldsymbol{\tau}_{11: t}, \boldsymbol{\tau}_{21: t} \ldots \boldsymbol{\tau}_{N_{1: t} 1: t}\right]^{\mathrm{T}}$ with the VTs. This is formally described by the Joint Probabilistic Data Association (JPDA) conditioned on the measured delays and the Inertial Measurement Unit (IMU) measurements $\mathbf{u}_{1: t}$ as

$$
\mathrm{p}\left(\mathbf{x}_{0: t}, \mathbf{n}_{1: t} \mid \mathbf{z}_{1: t}, \mathbf{u}_{1: t}\right)
$$

where the system state consists of a user system state and a VT system state as

$$
\mathbf{x}_{0: t}=\left[\mathbf{x}_{\mathrm{u}, 0: t}^{\mathrm{T}}, \mathbf{x}_{\mathrm{v}, 1: L 0: t}^{\mathrm{T}}\right]^{\mathrm{T}} .
$$

The PDF (2) can be approximated using PF [15] with particle weights calculated sequentially as

$$
w_{t \mid t-1}^{(k)}=w_{t}^{(k)} w_{t-1 \mid t-2}^{(k)},
$$

where

$$
w_{t}^{(k)} \propto \mathrm{p}\left(\mathbf{z}_{t} \mid \mathbf{x}_{\mathrm{u}, t}^{(k)}, \mathbf{z}_{t-1}\right)
$$

and

$$
w_{t-1 \mid t-2}^{(k)} \propto \operatorname{bel}\left(\mathbf{n}_{1: t-1}\right) \operatorname{bel}\left(\mathbf{x}_{\mathrm{u}, 0: t-1}\right),
$$

with

$$
\begin{aligned}
\operatorname{bel}\left(\mathbf{n}_{1: t}\right) \operatorname{bel}\left(\mathbf{x}_{\mathrm{u}, 0: t}\right)= & \eta \mathrm{p}\left(\mathbf{z}_{t} \mid \mathbf{n}_{t}, \mathbf{x}_{t}\right) \mathrm{p}\left(\mathbf{n}_{t} \mid \mathbf{n}_{t-1}\right) \\
& \operatorname{p~}\left(\mathbf{z}_{t} \mid \mathbf{x}_{\mathrm{u}, t}, \mathbf{z}_{t-1}\right) \mathrm{p}\left(\mathbf{x}_{\mathrm{u}, t} \mid \mathbf{x}_{\mathrm{u}, t-1}, \mathbf{u}_{t}\right) \\
& \operatorname{bel}\left(\mathbf{n}_{1: t-1}\right) \operatorname{bel}\left(\mathbf{x}_{\mathrm{u}, 0: t-1}\right),
\end{aligned}
$$

defining the recursive belief functions assuming a first-order Hidden Markov Model (HMM), and using Bayes' rule.

Finally, the transmitter position estimation is given by the set of kernels $\mathbf{x}_{\mathrm{v}, \ell t}^{(k p)}$ weighted by $w_{\ell t \mid t-1}^{(k p)}$ defining a multivariate PDF. This approach is known as a Regularized Particle Filter (RPF) [15].

\section{Sequential Estimation of the Gaussian Mixture Model Parameters}

As the Channel-SLAM algorithm is implemented as a sequential estimator of a user position, we require the VT state posterior to be estimated sequentially to evaluate (5). The approach in [10] achieves this by modeling the VT using an RPF.

The initial set of VT samples is obtained by sampling from a prior PDF. The prior PDF of $\mathrm{VT}_{\ell}$ can be visualized as a right cone surface with the base radius and height equal to the measured delay $z_{i t}$ of $\mathrm{MPC}_{i}$. This can be written as

$$
\left\|\mathbf{x}_{\mathrm{Rx}, t}, \mathbf{x}_{\mathrm{Tx}, \ell t}\right\|^{2}=\left(z_{i t}-b_{\mathrm{u}, t}-b_{\mathrm{v}, \ell t}\right)^{2},
$$

where $\mathbf{x}_{\mathrm{Rx}, t}$ and $\mathbf{x}_{\mathrm{Tx}, \ell t}$ represent $2 \mathrm{D}$ Cartesian coordinates of a user position and a VT position, respectively. The $z_{i t}$ is a measured distance obtained by dividing the speed of light by the measured delay of $\mathrm{MPC}_{i}, b_{\mathrm{u}, t}$ is an additional propagation distance associated with a receiver time offset, and $b_{\mathrm{v}, \ell t}$ is an additional propagation distance associated with a VT. For the initialization step, the set of VT samples is obtained by drawing samples uniformly from the right cone defined by (8), where $z_{i t}$ is a normally distributed random variable with variance corresponding to MPC delay estimator variance.

To sufficiently approximate this prior PDF requires many samples proportional to the measured delay square and inversely proportional to the measurement variance. The number of weights is given by the number of particles in all of the VTs. The weight calculation presents the position algorithm's main computational load. Hence, to reduce the computational load, we need to reduce the number of particles while preserving position estimation precision. 


\section{A. Modeling Virtual Transmitter with a Gaussian Mixture Model}

Similarly to the RPF [15], we draw particles from the continuous PDF given by a Gaussian mixture approximating the VT posterior

$$
\mathrm{p}\left(\mathbf{x}_{\mathrm{v}, \ell t}\right) \approx \sum_{p=1}^{P} w_{\ell t \mid t-1}^{(p)} \mathcal{N}\left(\mathbf{x}_{\mathrm{v}, \ell t}-\mathbf{x}_{\mathrm{v}, \ell t}^{(p)}, \Sigma_{\ell t}\right),
$$

which is a definition of the GMM in [14] with weights of individual GMM components corresponding to $w_{\ell t \mid t-1}^{(p)}$.

To see this approach's advantage, we need to discuss the approximation error caused by using samples from the kernels as proposed in RPF. While performing the RPF resampling step, each particle is treated as a PDF distributed according to the same kernel. The kernel is estimated according to the particle covariance matrix estimation

$$
\hat{\Sigma}_{\mathbf{x}_{\mathrm{v}, \ell t}^{(k)}}=\sum_{p} w_{\ell t \mid t-1}^{(k p)}\left(\mathbf{x}_{\mathrm{v}, \ell t}^{(k p)}-\hat{\mu}_{\mathbf{x}_{\mathrm{v}, \ell t}^{(k)}}\right)\left(\mathbf{x}_{\mathrm{v}, \ell t}^{(k p)}-\hat{\mu}_{\mathbf{x}_{\mathrm{v}, \ell t}^{(k)}}\right)^{\mathrm{T}}
$$

where the estimated mean is given by

$$
\hat{\mu}_{\mathbf{x}_{\mathrm{v}, \ell t}^{(k)}}=\sum_{p} w_{\ell t \mid t-1}^{(k p)} \mathbf{x}_{\mathbf{v}, \ell t}^{(k p)}
$$

then the new set of samples is obtained from a set of kernels placed to each particle's position.

Since the actual covariance is nonlinearly dependent on the $\mathbf{x}_{\mathrm{Rx}, t}^{(k)}$, and $\mathbf{x}_{\mathrm{Tx}, \ell t}^{(k p)}(10)$ provides a poor approximation of the actual covariance in the particle vicinity. The sample weights obtained by this method will almost surely diminish. This is especially true in the early stages after the VT initialization when the PDF is strongly multi-modal. In the multi-modal case, the RPF requires to decrease the kernel width [15], which in the Channel-SLAM means to assure the kernel width is comparable to an observation covariance.

From the other point of view, if the new samples are obtained using the Gaussian kernel, the VT particles perform the Brownian movement during the resampling step. This contradicts the assumption used during the Channel-SLAM derivation [8]-[10], where the VT has to be fixed. The only way how to use the RPF for the VT state estimation is to decrease the kernel width that, from the user movement point of view, VT particles seem fixed.

On the other hand, the proposed GMM-based algorithm assumes a fixed position of GMM components assuring the fixed VT position. We perform the posterior PDF exploration by creating new GMM components drawn from the actual posterior PDF estimation represented by the GMM. The exploration step allows using a lower number of GMM components during the VT initialization step. The implementation of the GMM resampling step, including the posterior PDF, exploration is described in the following section.

\section{B. Implementation}

The difference of the state-of-the-art implementation of the Channel-SLAM [10] and the proposed method is in the VT state PF's resampling step.
Similarly to [10], we use the systematic resampling scheme [15] to obtain a set of new GMM components by drawing the components with the probability corresponding to its weights $w_{\ell t \mid t-1}^{(p)}$. However, during the systematic resampling step, we do not draw the components multiple times. The proposed algorithm draws each of the components not more than ones and assigns it with a weight corresponding to the required occurrences. Hence, the obtained PDF represents the same PDF as the systematic resampled one while using a lower number of components.

The key idea is to reuse the resources preserved by resampling without repetition to improve the PDF approximation and reduce the PF's degeneracy phenomenon. Sampling $P_{\text {add }}$ samples from the GMM with $P_{\text {unq }}$ unique components obtained during the resampling without repetition provides exploration samples. Assigning the $P_{\text {add }}$ samples with a uniform weight, appending with $P_{\text {unq }}$ components, and normalizing the new set of $P=P_{\text {add }}+P_{\text {unq }}$ weights produces a new GMM approximating the VT PDF.

Similarly to RPF, we use a fraction of estimated covariance (10) as a covariance for each GMM component. However, since the initialization of the $\mathbf{x}_{\mathrm{v}, \ell t}^{(k)}$ according to (8) is a multimodal PDF, directly using the estimated covariance would produce overly pessimistic samples with a close to zero likelihood. This effect is particularly severe during the early iterations.

To reuse the released resources most efficiently, we desire to explore areas with most of the PDF mass and not waste limited resources within a close to zero probability area. We first sample position components from a GMM while ignoring the $b_{\mathrm{v}, \ell t}$ axis, and its correlations with position components to achieve this goal. Then we assign a $b_{\mathrm{v}, \ell t}^{\prime}$ value according to

$$
b_{\mathrm{v}, \ell t}^{\prime}=b_{\mathrm{v}, \ell t}+\left\|\mathbf{x}_{\mathrm{Rx}, t}, \mathbf{x}_{\mathrm{Tx}, \ell t}\right\|-\left\|\mathbf{x}_{\mathrm{Rx}, t}, \mathbf{x}_{\mathrm{Tx}, \ell t}^{\prime}\right\|+v,
$$

where the apostrophe marks value obtained from a corresponding GMM component, and $v$ is a zero-mean normally distributed additional value.

We claim that this method provides a set of new samples that, on average, have the same likelihood as the GMM component from which were the samples obtained. This can be easily proven by setting

$$
\mathbf{E}\left[\frac{\mathrm{p}\left(\mathbf{z}_{t}\right.}{\left.\mathbf{x}_{\mathrm{u}, t}^{(k)}, \mathbf{x}_{\mathrm{Tx}, \ell t}, b_{\mathrm{v}, \ell t}\right)}\right]=1,
$$

where the expectation is taken with respect to $b_{\mathrm{v}, \ell t}^{\prime}$ and the observation likelihood is defined as

$$
\begin{aligned}
& \mathrm{p}\left(\mathbf{z}_{t} \mid \mathbf{x}_{\mathrm{u}, t}^{(k)}, \mathbf{x}_{\mathrm{v}, \ell t}^{(k p)}\right)= \\
& \frac{1}{\sqrt{2 \pi Q}} \mathrm{e}^{-\frac{\left(z_{i t}-\left\|\mathbf{x}_{\mathrm{Rx}, t}^{(k)}, \mathbf{x}_{\mathrm{Tx}, \ell t}^{(k p)}\right\|-b_{\mathbf{u}, t}^{(k)}-b_{\mathbf{v}, n_{i t}^{(k)} t}^{(k p)}\right.}{2 Q}} .
\end{aligned}
$$

Additionally, one can notice that the likelihood function motivates the initialization using (8) because the maximum likeli- 


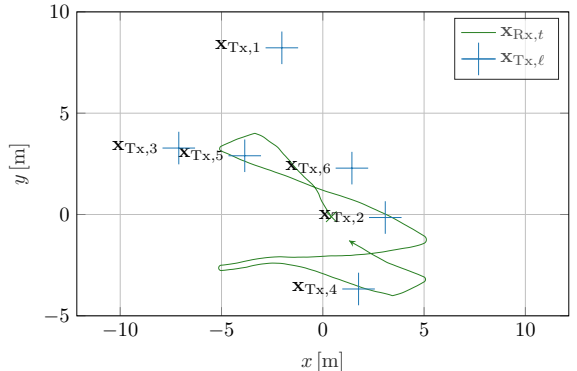

Fig. 2. The example of the simulated trajectory shown in green with the marked beginning and the end. The randomly generated VTs are marked with blue crosses, where the $\mathbf{x}_{\mathrm{Tx}, 1}$ is the physical transmitter. The figure shows the RMSE curves for two different particle volumes.

hood for a fixed position $\mathbf{x}_{\mathrm{Rx}, t}^{(k)}$, and the additional propagation distances $b_{\mathrm{u}, t}^{(k)}$, and $b_{\mathrm{v}, \ell t}$, defines a right cone.

\section{EVALUATIONS}

To show the advantage of the presented method, we compare it with the state-of-the-art approach. [10] We use simulations of the band-limited Ultra-WideBand (UWB) channel. Each simulation assumes one physical transmitter and five reflections. The White Noise Acceleration (WNA) movement model [17] simulates the user trajectory. We assume that the received ranging waveform is processed by a channel parameter estimator approaching Cramer-Rao Lower bound (CRLB). The noisy delay estimates serve as an input for the algorithms. Finally, we assume outages of the MPC tracking occurring when the Rayleigh distributed random amplitude of the MPC is lower than some threshold. We use $3 \mathrm{~dB}$ above the noise level as a threshold.

The evaluation compares the proposed algorithm with the state-of-the-art approach for different amounts of particles used to initialize the VTs. The simulations are performed for ten randomly generated scenarios. An example of a randomly generated scenario is shown by Fig. 2, where the user is walking inside a rectangular room for 100 seconds. Due to the simulation and evaluation randomness, all of the evaluations are repeated ten times.

Throughout the evaluations, the RMSE is used to compare the algorithm performance. Since each VT havs a different propagation loss, the delay estimation variances differ significantly. The evaluated RMSE for each VT is normalized by its delay Standard Deviation (STD) to show the VT estimator's performance. The user and VT position estimation performance over time is shown in Fig. 3a and Fig. 3b, respectively. The plots show two different amounts of simulated particles. The particle set size is defined by the particles needed to approximate the user state; however, it also depends on the MPC delay during the initialization and its variance Hence, the number of particles varies between $1 \times 10^{4}<N_{\text {particles }}<5 \times 10^{6}$. The different amounts of particles for individual simulations are obtained by changing one multiplicative constant influencing the number of particles used to initialize VT.

We assume that the initial user state is known. Hence, the initial RMSE in Fig. 3a starts at zero and then slowly increases

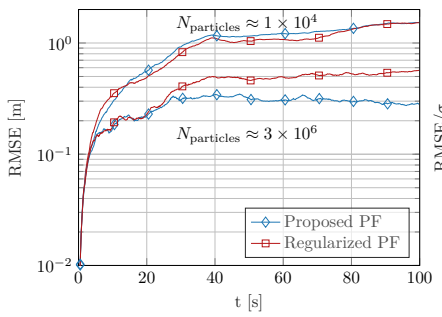

(a)

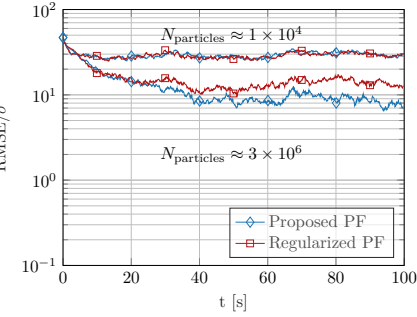

(b)
Fig. 3. The RMSE of the position $\mathbf{x}_{\mathrm{Rx}, t}$ (a) and VT position (b) estimated using the proposed PF resampling method marked by a solid blue line, and the state-of-the-art Channel-SLAM algorithm shown by dashed red line. Each VT is normalized by the corresponding STD to be able to plot one generalizing RMSE curve for the VTs with different observation covariance. The figures show the RMSE curves for two different particle volumes.

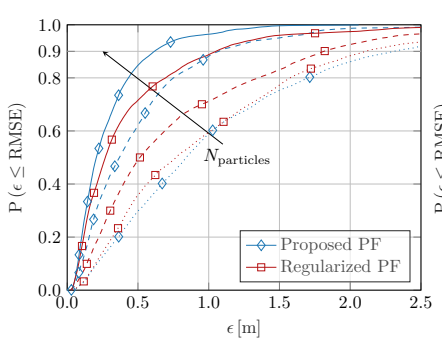

(a)

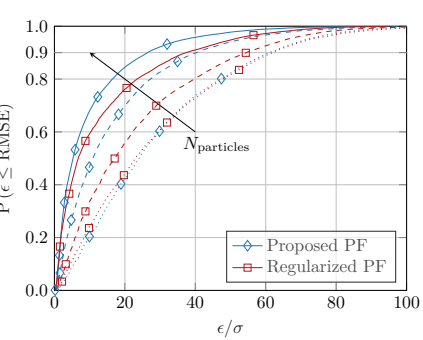

(b)
Fig. 4. The CDF of the position $\mathbf{x}_{\mathrm{Rx}, \mathrm{t}}$ (a) and VT position (b) RMSE estimated using the proposed PF resampling method marked by a solid blue line, and the state-of-the-art Channel-SLAM algorithm shown by dashed red line. Each VT is normalized by the corresponding STD to be able to plot one generalizing RMSE curve for the VTs with different observation covariance. The figures show the RMSE curves for three different particle volumes.

because the VTs are not yet estimated well enough to correct the user state prediction error. On the other hand, the VTs are initialized uniformly within the whole prior PDF given by (8). Hence, in Fig. 3b, the VT RMSE is maximal initially and decreases over time.

The plotted Cumulative Distribution Functions (CDFs) in Fig. 4 shows better performance improvement. The additional dashed plot shows the performance when the number of VT particles is increased ten times than the minimum $N_{\text {particles }} \approx 1 \times 10^{4}$. While the state-of-the-art method still performs poorly, the proposed method is already comparable with the best precision achievable by the state-of-the-art algorithm.

To fully capture the sensitivity of the compared algorithms

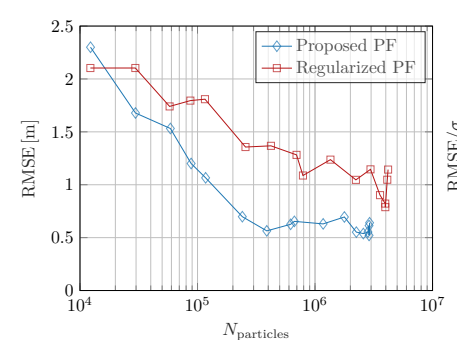

(a)

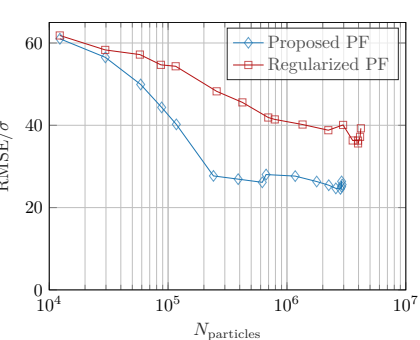

(b)
Fig. 5. The comparison of the proposed and the state-of-the-art PF resampling method RMSE achieved for a given number of particles. The $90 \%$ confidence intervals of RMSE for a user (a) and VT (b) position are plotted. 
concerning the number of particles, we show, in Fig. 5, the $90 \%$ confidence interval of the RMSE achievable for a given number of particles. Fig. 5b shows that for an extremely low number of VT particles, the RMSE converges to a common point. The lowest point is using approximately ten particles per VT per user particle.

With the number of particles this low, the probability of converging to a correct VT position is marginal. However, even for the lowest number of particles, the measurements help estimate the user position better. When tracking the user position using only the movement model, the RMSE is more than twice the RMSE $<2.5 \mathrm{~m}$ achieved using VT with the lowest number of particles.

As we increase the number of particles, the RMSE is decreasing faster for the proposed algorithm than the stateof-the-art approach, which is achieved thanks to the reusing the resources, redeemed during the resampling step, to explore the areas where the likelihood is assumed to be large. The proposed algorithm is allowing to obtain the assumed maximal achievable precision for orderly less number of particles. The scenario itself, together with the VT placement and the user trajectory, limits the achievable precision, making calculating the true maximal achievable precision particularly hard. We assume achieving the maximal achievable precision when the RMSE is no longer improving with an increasing number of particles.

Interestingly, the proposed method can achieve better precision even for a high number of particles, where both algorithms would be expected to converge to the same precision. This might be caused by the fact that the VT in the state-ofthe-art approach performs a weak Brownian movement during the kernel resampling step. While our GMM-based method assumes fixed VTs as required by the Channel-SLAM algorithm and performs exploration to approximate the posterior PDF using a low number of particles.

The VT precision effects the RMSE of the user state estimation depicted by Fig. $5 \mathrm{a}$ in the same manner.

\section{CONCLUSION}

The proposed GMM-based Channel-SLAM algorithm significantly improves the state-of-the-art multipath assisted positioning approach. The evaluation shows that the proposed method allows decreasing the number of particles more than 10 times while preserving the same precision as the stateof-the-art approach. Not only that, the memory consumption is significantly reduced, thanks to the proposed resampling approach, the computational complexity is also reduced. This was achieved by releasing and reusing resources during the $\mathrm{PF}$ resampling schedule. Finally, increasing the number of particles, the GMM-based algorithm converges to a lower RMSE than the RPF-based state-of-the-art approach. A possible explanation is that the GMM-based model assumes a fixed VT state in time, while the RPF-based state-of-the-art approach performs Brownian movement violating one of the Channel-SLAM assumptions.

\section{ACKNOWLEDGMENT}

This work has been performed in the framework of the CTU grant SGS19/168/OHK3/3T/13 Electromagnetic structures and waves and the DLR project Navigation 4.0.

\section{REFERENCES}

[1] P. Bahl and V. N. Padmanabhan, "Radar: An in-building rf-based user location and tracking system," in Proc. IEEE INFOCOM, Tel-Aviv, Israel, 2000, pp. 775-784 vol.2.

[2] E. Kupershtein, M. Wax, and I. Cohen, "Single-site emitter localization via multipath fingerprinting," IEEE Trans. Signal Process., vol. 61, no. 1, pp. 10-21, Jan. 2013, 10.1109/tsp.2012.2222395.

[3] T. Deissler and J. Thielecke, "Feature based indoor mapping using a bat-type UWB radar," in Proc. ICUWB, Vancouver, BC, Canada, 2009, pp. 475-479.

[4] M. Froehle, E. Leitinger, P. Meissner, and K. Witrisal, "Cooperative multipath-assisted indoor navigation and tracking (co-MINT) using UWB signals," in Proc. ICC, Atlanta, GA, USA, 2013, pp. 16-21.

[5] P. Meissner, E. Leitinger, and K. Witrisal, "UWB for robust indoor tracking: Weighting of multipath components for efficient estimation," IEEE Wireless Commun. Lett., vol. 3, no. 5, pp. 501-504, Oct. 2014, 10.1109/lwc.2014.2341636.

[6] T. Deissler and J. Thielecke, "UWB SLAM with rao-blackwellized monte carlo data association," in Proc. IPIN, Zurich, Switzerland, 2010, pp. 1-5.

[7] E. Leitinger, F. Meyer, F. Tufvesson, and K. Witrisal, "Factor graph based simultaneous localization and mapping using multipath channel information," in Proc. ICC Workshops, Paris, France, 2017, pp. 652-658.

[8] C. Gentner, T. Jost, W. Wang, S. Zhang, A. Dammann, and U.-C. Fiebig, "Multipath assisted positioning with simultaneous localization and mapping," IEEE Trans. Wireless Commun., vol. 15, no. 9, pp. 6104 6117, Sep. 2016, 10.1109/twc.2016.2578336.

[9] C. Gentner, R. Pöhlmann, M. Ulmschneider, T. Jost, and S. Zhang, "Positioning using terrestrial multipath signals and inertial sensors," Mobile Information Systems, vol. 2017, pp. 1-18, Oct. 2017, $10.1155 / 2017 / 9170746$.

[10] R. Karásek and C. Gentner, "Stochastic data association for multipath assisted positioning using a single transmitter," IEEE Access, vol. 8, pp. 46 735-46 752, 2020, 10.1109/ACCESS.2020.2977558.

[11] A. Doucet, N. d. Freitas, K. P. Murphy, and S. J. Russell, "Raoblackwellised particle filtering for dynamic bayesian networks," in Proc. UAI, San Francisco, CA, USA, 2000, pp. 176-183.

[12] M. Ulmschneider, C. Gentner, T. Jost, and A. Dammann, "Raoblackwellized gaussian sum particle filtering for multipath assisted positioning," Journal of Electrical and Computer Engineering, vol. 2018, pp. 1-15, Apr. 2018, 10.1155/2018/4761601.

[13] E. B. Sudderth, A. T. Ihler, M. Isard, W. T. Freeman, and A. S Willsky, "Nonparametric belief propagation," Communications of the $A C M$, vol. 53, no. 10, pp. 95-103, Oct. 2010, 10.1145/1831407.1831431.

[14] D. Alspach and H. Sorenson, "Nonlinear bayesian estimation using gaussian sum approximations," IEEE Transactions on Automatic Control, vol. 17, no. 4, pp. 439-448, Aug. 1972.

[15] M. Arulampalam, S. Maskell, N. Gordon, and T. Clapp, "A tutorial on particle filters for online nonlinear/non-gaussian bayesian tracking," IEEE Trans. Signal Process., vol. 50, no. 2, pp. 174-188, Aug. 2002, 10.1109/78.978374.

[16] P. Bello, "Characterization of randomly time-variant linear channels," IEEE Trans. Commun. Syst., vol. 11, no. 4, pp. 360-393, Dec. 1963 10.1109/TCOM.1963.1088793.

[17] Y. Bar-Shalom, X.-R. Li, and T. Kirubarajan, Estimation with Applications to Tracking and Navigation: Algorithms and Software for Information Extraction, 1st ed. New York, NY, USA: John Wiley \& Sons, Inc., 2001. 